\title{
A Note on Nonemptiness of Cognitive Semantics for Linguistic Representations of Modal Equivalence
}

\author{
R. Katarzyniak, D. Więcek \\ Wroclaw University of Science and Technology \\ Faculty of Computer Science and Management, Department of Informatics \\ Laboratory of Computational Semiotics and Interactive Systems \\ E-mail: \{radoslaw.katarzyniak,dominik.wiecek\}@pwr.edu.pl
}

Received: 10 October 2018; revised: 07 December 2018; accepted: 07 December 2018; published online: 28 December 2018

\begin{abstract}
In this paper we deal with an original model of technically oriented cognitive semantics of modal equivalences, stated in semi-controlled natural languages and used as surface linguistic representations of internal knowledge states of artificial autonomous systems. The cognitive semantics is defined to support generation of natural language statements extended with auto-epistemic operators of modality, representing subjectively experienced certainty, strong belief and week belief of the autonomous system that the relationship between occurrence of two chosen properties in a real object is the logical equivalence relation. An internal architecture of the autonomous system is briefly presented with particular emphasis on computational modules responsible for verification of compliance of current knowledge processing states with states described by the cognitive semantics as proper for application of a particular case of modal equivalence. The major issue discussed in the paper concerns an original theory of grounding of modal equivalences in empirical knowledge resources, encapsulated in the autonomous system. The whole theory consists of theorems describing basic and advanced properties of the cognitive semantics. In the paper, the attention is limited to presentation and proof of four theorems about nonemptiness of the proposed cognitive semantics. The research results cover the next stage of some research and development efforts, concerning the technical implementation of cognitive computing models, describing the artificial generation of semi-natural languages by the interactive autonomous system to describe their encapsulated knowledge resources.
\end{abstract}

Key words: linguistic representation, natural language connective, equivalence, autoepistemic modality, natural language engineering, artificial cognitive system, cognitive computing

\section{INTRODUCTION}

In paper [10] an original model of cognitive semantics of modal equivalences used as surface linguistic summaries was presented. The considered summaries were assumed to be built by an autonomous system of knowledge using language-specific surface representations of the logical sense of an equivalence functor, names of two different features for which their logical coexistence in an actual object was considered as the main issue communicated by the summaries, and three autoepistemic modal operators, each corresponding to one of three distinguishable levels of confidence of the autonomous system as to the consistency of the sense of the generated summary with the real state of the world. It was assumed that distinguishing between three levels of knowledge confidence was a purely subjective cognitive ability of the autonomous system using modal linguistic summaries as surface representations of its knowledge. All the modal equivalences studied in paper [10] and [34] are further listed and commented on in more detail in the next section hereof (see Tables 1 and 2).

In the cited work [10] a preliminary version of cognitive semantics of modal equivalences was presented and its verification was carried out by simulation research, to illustrate convergence of artificial linguistic behaviors based on 
the proposed cognitive semantics with linguistic behaviors of the subject of knowledge using a natural language. The conducted simulations showed that in the scope of cognitive behavior covered by the proposed definition, the model of cognitive semantics met the common sense assessment of its pragmatic commonsense coherence and correctly summarized the content of the used test collections, taking into account the level of data processing available to the autonomous system producing the summaries.

In this article, we present the next stage of research work related to the construction of technically oriented cognitive semantics of the considered class of modal sentences. The new theoretical elements, in relation to the results presented in [10], are as follows:

- the original extension of the cognitive semantics of modal equivalence to a form involving the case of the cognitive state occurring in the autonomous system before initiating the process of extracting modal equivalence, and

- proof of the basic theorem of the original theory of grounding of modal equivalences, stating that the proposed version of cognitive semantics is not empty.

Consideration of the state of the autonomous system prior to initiation of extraction processes for linguistic representations of modal equivalence extends the scope of internal cognitive states of the autonomous artificial system for which surface linguistic representations are assigned in the model.

In turn, proving the non-emptiness of the proposed definitions of cognitive semantics of modal equivalence is the first step in constructing the original theory of grounding of modal equivalences in information resources stored in autonomous artificial systems and ultimately demonstrating the convergence of artificial behaviors based on proposed cognitive semantics with natural language behaviors, obviously in the processing of natural language sentences communicating the sense of logic equivalence.

The order of the article is as follows. In the second section, the class of considered modal equivalences and symbolic representation adopted for them is presented in more detail. In the third section, for completeness of the presentation, the model of knowledge bases of an autonomous system is invoked in order to precisely point at states of their processing which are covered by the given cognitive semantics of modal equivalences. Other important elements of the cognitive system are also presented, participating in the formulation of a technically oriented definition of this semantics. The fourth section presents a complete definition of cognitive semantics. In the fifth section, the basic theorem of the original theory of grounding of modal equivalences in the cognitive states of the described autonomous system with the proof of this theorem is formulated. The comments section summarizes the results presented earlier.

\section{A CLASS OF AUTOEPISTEMIC EQUIVALENCES AND THEIR PRAGMATICS}

As it has been stated above the analysis of theoretical properties, proposed in the further parts of the article, concerns the original cognitive semantics describing the logical meaning that at the natural language level can be communicated with the sentences listed in Table 2 . The cognitive semantics, as considered in this paper, is strongly related to the cognitive phenomenon of symbol grounding [25, 29, 33, 36], as well as the symbol anchoring [2,32], considered in the context of semi-natural (controlled) and natural languages. The basic definition of symbol grounding and anchoring assumes that these phenomena rely on the binding of language symbols to the observations of real objects of the world. Obviously, in the case of living systems the implementation of the bond takes place with direct and indirect participation of a number of specialized cognitive structures, cognitive processes (both deep and shallow) and internal mental states of autonomous living systems. For individual language symbols these elements, i.e. structures, processes and mental states, are used in models of their cognitive semantics. The considered modal statements are an example of such symbols.

There are two issues related to the list of modal sentences given in Table 2 worth emphasizing in the current research context.

At first, as it appears from the form of sentences in Table II, it is assumed for this research that the commonsense interpretation of considered modal equivalences is communicated in the autoepistemic perspective, to stress the subjective nature of knowledge being communicated. In general, special features and main differences between epistemic and autoepistemic modes of interpreting linguistic descriptions of knowledge states were discussed in detail elsewhere, e.g. [9] (see Part II - Grounding and BDI models).

From the point of view of these studies, it is worth emphasizing three of the specific features of the autoepidememic perspective:

- the auto-epistemic perspective is essentially correlated with the intention of the subject of knowledge, i.e. the autonomous system, to generate messages representing the subject's own experience of the fact that the logical equivalence relationship occurs between observing property $\mathrm{P}$ and property $\mathrm{Q}$, in an object of a real world,

- adoption of the auto-epistemic perspective of the description of the states of knowledge is signaled at the level of surface linguistic representation through the use of the pronoun "I" as the subject.

- an attempt to construct a technically oriented model of auto-epistemic perspective naturally excludes the possibility of applying the idea of classical semantics as the theoretical basis of language generation proce- 
dures; classical semantics must be replaced by cognitive semantics $[22,30]$, and the aim of theoretical research is to formulate and verify the case of this semantics related to the logical sense of modal equivalences.

At second, the main objects of interest in this research are the sentences belonging to the class of modal equivalence, constructed using exactly three modal operators Know, Bel and Pos. The choice of three modal operators, instead of two, distinguishes the model presented in this article from other popular models of knowledge states description, proposed by other authors.

Namely, in most source models of knowledge states, providing a starting point for many subsequent models, only two dual operators of modality $\square$ and $\diamond$ corresponding to cognitive experience of certainty (knowledge) and belief, respectively, and used to extend non-modal formulas were subject to some theoretical analysis, e.g. see models presented in $[1,3-5,14,18,19,24,27]$.

Obviously, the choice of three operators as the linguistic extensions of non-modal representation of the logical sense of equivalence is not accidental and results from the pragmatics of natural languages. Indeed, in natural languages, three groups of surface linguistic markers corresponding to the following three cognitively distinguished ranges of certainty ascribed to the subjective beliefs of the autonomous system are usually taken into account:

- certainty of the communicated state of affairs to be actually realized (corresponding to the concept of knowledge about the state),

- strong conviction (belief) about the communicated state of affairs to be actually realized (corresponding to the internal experience of the high probability of the current realization of this state), and

- low conviction (belief) about the communicated state of affairs to be actually realized (corresponding to the internal experience of relatively low probability of the current realization of this state).

Obviously, in this research, the state of affairs being evaluated as to the certainty of its actual occurrence is the cognitive experience identified with the logical sense of the equivalence.

The definition of technically oriented cognitive semantics and its implementation in autonomous computing systems is in general a difficult task. It involves the design and implementation of technical equivalents of a number of complex structures of knowledge representation and cognitive processes responsible for building and maintaining relations between information resources encapsulated in the knowledge subject and surface (linguistic) representations of these resources (or selected fragments of these resources).

Several earlier works have already proposed technically oriented cognitive semantics for the following classes of modal sentences used for external (surface) representation of internal (encapsulated) states of knowledge:

- simple modalities, e.g. [6, 9],

- modal conjunctions, e.g. [12, 13, 23],

- modal exclusive and inclusive alternatives, e.g. [9, 12], and

- modal conditionals, e.g. [15, 26].

These semantics were verified analytically and empirically in terms of their compliance with the predefined set of commonsense postulates determining the generation of linguistic summaries of knowledge in natural languages statements.

The shared element of the above mentioned semantics is found in the same set of functional modules and similar encapsulated classes of knowledge representation structures. This set of modules and structures is also considered for the case of generating modal equivalences and includes:

- a dedicated knowledge base designed to store knowledge of an empirical nature, acquired by the subject of knowledge during interaction with the real world,

- a dedicated knowledge base for gathering knowledge of a semantic nature, including representations of the results of the current processing of the contents of the empirical knowledge base;

- a specialized interface, which for a given case of modal representations of knowledge is responsible for the technical implementation of the cognitive semantics function. This interface links the repository, covering the base of empirical knowledge (episodic) and the base of semantic knowledge and the degree of processing of this repository with surface linguistic representations described by the cognitive semantics proposed for them.

What is more, the interface is defined for each semantics, including the so-called system of modality thresholds - an original tool for verifying the possibility of using a specific modal operator in the existing state of knowledge processing as a linguistic marker extending the surface representation of particular logic sense.

For the completeness of the presentation of the cognitive semantics model proposed herein, it is worth mentioning the notion of linguistic protoform. In literature, the term protoform can cover both individual modal formulas listed in table 1 (e.g. $\operatorname{Know}(p(o) \Leftrightarrow q(o)), \operatorname{Bel}(p(o) \Leftrightarrow q(o))$, $\operatorname{Pos}(p(o) \Leftrightarrow q(o)))$, as well as their symbolic generalizations (e.g. $\Omega(p(o) \Leftrightarrow q(o)), \Omega(p(o) \Leftrightarrow \neg q(o)), \Omega(\neg p(o) \Leftrightarrow$ $q(o)), \Omega(\neg p(o) \Leftrightarrow \neg q(o))$ ) (see [10, 34]). In general, a linguistic protoform is usually understood as a formal structure which, at its specific level of abstraction, represents the concrete order (specific state of transformation) of the subject's private knowledge, specific to the group of linguistic representations embraced by this protoform, understood as alternative representations of the same logical sense.

Elements of the autonomous computing system, which have been briefly described in this section, will be formally 
Tab. 1. Natural language representation of logical sense of non-modal equivalences

\begin{tabular}{l|l}
\hline Formula & Commonsense meaning in natural language \\
\hline \hline$p(o) \Leftrightarrow q(o)$ & $\begin{array}{l}\text { [According to all that I have experienced (as subject A) (I can state what follows :)] } \\
\text { Object o exhibits property } P \text { if and only if it [the object o] exhibits property } Q .\end{array}$ \\
\hline$p(o) \Leftrightarrow \neg q(o)$ & $\begin{array}{l}\text { [According to all that I have experienced (as subject A) (I can state what follows :)] } \\
\text { Object o exhibits property } P \text { if and only if it [the object o] does not exhibit property } Q .\end{array}$ \\
\hline$\neg p(o) \Leftrightarrow q(o)$ & $\begin{array}{l}\text { [According to all that I have experienced (as subject A) (I can state what follows :)] } \\
\text { Object o does not exhibit property } P \text { if and only if it [the object o] exhibits property } Q .\end{array}$ \\
\hline$\neg p(o) \Leftrightarrow \neg q(o)$ & $\begin{array}{l}\text { [According to all that I have experienced (as subject A) (I can state what follows :)] } \\
\text { Object o does not exhibit property } P \text { if and only if it [the object o] does not exhibit } \\
\text { property } Q .\end{array}$ \\
\hline
\end{tabular}

defined in the next section, obviously for the case of the artificial autonomous system with the ability to generate surface representations listed in Tabs. 1 and 2.

\section{A MODEL OF ARTIFICIAL AGENT AND AUTONOMOUS COGNITIVE STATE}

\section{1. Repository of episodes and state of episodic knowledge processing}

We further assume that the external environment of the autonomous system consists of a set of atomic objects $o \in O$. Each object from the environment can either exhibit or not exhibit a property $P \in \Delta$, where $\Delta=\left\{P_{1}, \ldots, P_{K}\right\}$ is a finite set of all observable properties. Individual world states are assumed to be always given in relation to individual discrete time points $t \in T$ and are represented by the following structure:

Definition 1. An individual state of external environment occurring at the time point $t$ is represented by the following encapsulated tuple:

$$
W P(t)=<O, P_{1}(t), \ldots, P_{K}(t)>
$$

where $P_{i}(t)$ represents a set of all objects that occur as exhibiting property $P_{i}$ at time point $t$.

Obviously, it follows from the closed-world assumption that the set $O / P_{i}(t)$ consists of all objects that do not exhibit property $P_{i}$ at time point $t$.

It is also assumed (and technically realized) in our research that the considered autonomous system is capable of perceiving the above introduced external environment through a set of dedicated sensors. As such, these sensors are a form of a direct interface between the autonomous system and the external world, and results of individual observations are encapsulated in an internal empirical knowledge base consisting of episodes. Such a knowledge base is a strictly private body of knowledge about the state of the external environment and consists of information regarding the state of different objects, belonging to the perceivable properties $P_{1}, \ldots, P_{K}$ and accessible in the environment to the sensory system at current time point $t$.

Data obtained from the external environment at the same situation and by the autonomous sensory system are integrated into a complex knowledge structure called episode which is an individual element of an empirical knowledge base. The concept of episode is the same in many respects as the concept of episodic knowledge items used in [28].

In order to formally capture all the above concepts the following definitions are introduced:

Definition 2. An individual episode of the environment, perceived by the autonomous system as the same situation (in the same state) of the environment, is represented by the following encapsulated tuple

$$
E(t)=<O, P_{1}^{+}(t), P_{1}^{-}(t), \ldots, P_{K}^{+}(t), P_{K}^{-}(t)>
$$

where:

$P_{k}^{+}(t)$ is a set consisting of all objects $o_{i} \in O$ that at time point $t$ were registered (experienced) by the autonomous system of knowledge as exhibiting property $P_{k}$,

$P_{k}^{-}(t)$ is a set consisting of all objects $o_{i} \in O$ that at time point $t$ were registered (experienced) by the autonomous system of knowledge as non-exhibiting property $P_{k}$.

The tuples $W P(t)$ and $E(t)$, introduced above, are related in an apparent way to knowledge complex structures, called elsewhere $t$-related world profiles (Eq.1) and $t$-related base-profile ( $t$-related episode) (Eq.2), respectively [6] [11].

The overall content of the episodic (empirical) knowledge base is further formally represented as the following collection:

Definition 3. The episodic knowledge base is always represented in relation to particular time points t and given as the following collection of individual episodes:

$$
\text { Episodes }(t)=\left\{E\left(t_{n}\right): t_{n} \in T \text { and } t_{n} \leq t\right\} .
$$

As aforementioned, the role of Episodes $(t)$ is crucial due to its ultimate function in the induction of subjective references to external, made by the autonomous system and 
Tab. 2. Natural language representation of logical sense of modal equivalences

\begin{tabular}{|c|c|}
\hline Formula & Commonsense meaning in natural language \\
\hline $\operatorname{Pos}(p(o) \Leftrightarrow q(o))$ & $\begin{array}{l}\text { [According to all that I have experienced (as subject A) (I can state what follows :)] } \\
\text { It is possible that object o exhibits property } P \text { if and only if it [the object o] exhibits } \\
\text { property } Q \text {. }\end{array}$ \\
\hline $\operatorname{Pos}(p(o) \Leftrightarrow \neg q(o))$ & $\begin{array}{l}\text { [According to all that I have experienced (as subject A) (I can state what follows :)] } \\
\text { It is possible that object o exhibits property } P \text { if and only if it [the object o] does not } \\
\text { exhibit property } Q \text {. }\end{array}$ \\
\hline $\operatorname{Pos}(\neg p(o) \Leftrightarrow q(o))$ & $\begin{array}{l}\text { [According to all that I have experienced (as subject A) (I can state what follows :)] It is } \\
\text { possible that object o does not exhibit property P if and only if it [the object o] exhibits } \\
\text { property } Q .\end{array}$ \\
\hline $\operatorname{Pos}(\neg p(o) \Leftrightarrow \neg q(o))$ & $\begin{array}{l}\text { [According to all that I have experienced (as subject A) (I can state what follows :)] It } \\
\text { is possible that object o does not exhibit property } P \text { if and only if it [the object o] does } \\
\text { not exhibit property } Q .\end{array}$ \\
\hline $\operatorname{Bel}(p(o) \Leftrightarrow q(o))$ & $\begin{array}{l}\text { [According to all that I have experienced (as subject A) (I can state what follows :)] I } \\
\text { belive that object o exhibits property } P \text { if and only if it [the object o] exhibits property } \\
Q .\end{array}$ \\
\hline $\operatorname{Bel}(p(o) \Leftrightarrow \neg q(o))$ & $\begin{array}{l}\text { [According to all that I have experienced (as subject A) (I can state what follows :)] I } \\
\text { believe that object o exhibits property } P \text { if and only if it [the object o] does not exhibit } \\
\text { property } Q \text {. }\end{array}$ \\
\hline $\operatorname{Bel}(\neg p(o) \Leftrightarrow q(o))$ & $\begin{array}{l}\text { [According to all that I have experienced (as subject A) (I can state what follows :)] I } \\
\text { believe that object o does not exhibit property P if and only if it [the object o] exhibits } \\
\text { property } Q \text {. }\end{array}$ \\
\hline $\operatorname{Bel}(\neg p(o) \Leftrightarrow \neg q(o))$ & $\begin{array}{l}\text { [According to all that I have experienced (as subject A) (I can state what follows :)] I } \\
\text { believe that object o does not exhibit property } P \text { if and only if it [the object o] does not } \\
\text { exhibit property } Q \text {. }\end{array}$ \\
\hline $\operatorname{Know}(p(o) \Leftrightarrow q(o))$ & $\begin{array}{l}\text { [According to all that I have experienced (as subject A) (I can state what follows :)] I } \\
\text { known that [I am certain that] object o exhibits property } P \text { if and only if it [the object } \\
\text { o] exhibits property } Q \text {. }\end{array}$ \\
\hline $\begin{array}{l}\text { Know }(p(o) \\
\neg q(o))\end{array}$ & $\begin{array}{l}\text { [According to all that I have experienced (as subject A) (I can state what follows :)] I } \\
\text { know that object o exhibits property } P \text { if and only if it [the object o] does not exhibit } \\
\text { property } Q \text {. }\end{array}$ \\
\hline $\begin{array}{l}\text { Know }(\neg p(o) \\
q(o))\end{array}$ & $\begin{array}{l}\text { [According to all that I have experienced (as subject A) (I can state what follows :)] I } \\
\text { know that object o does not exhibit property } P \text { if and only if it [the object o] exhibits } \\
\text { property } Q .\end{array}$ \\
\hline $\begin{array}{l}\operatorname{Know}(\neg p(o) \\
\neg q(o))\end{array}$ & $\begin{array}{l}\text { [According to all that I have experienced (as subject } A \text { ) (I can state what follows :)] It } \\
\text { know that object o does not exhibit property } P \text { if and only if it [the object o] does not } \\
\text { exhibit property } Q \text {. }\end{array}$ \\
\hline
\end{tabular}

given in a form of linguistically oriented models, stored in the higher level semantic memory. Such references are necessary and especially important in situations when direct world's observation is restricted or impossible for some reasons.

In each state of the system (defined by the time point $t \in T$ ) agent's empirical knowledge is computationally divided into two parts. The first, called shallow, represents a conscious level of knowledge processing and is represented by a portion of already processed episodes from Episodes $(t)$. The second, called deep, represents the rest of the empirical material, which at a particular time point is beyond the reach of conscious cognition.

As it has already been mentioned, such a division received strong empirical justification and was quite well described in the cognitive science literature where the influence of deep levels of cognition was proved a crucial and not to be eliminated factor of natural cognitive behaviour, including natural language interpretation and production [31]. Moreover, it was also included in at least some of the computationally oriented models of natural language semantics and pragmatics, see the concepts of focus of attention in [16, 17] 
and centre of attention in [35].

In order to formally capture the concepts of deep and shallow portions of the empirical knowledge base, at least at the general level of understanding these concepts, two additional symbols are further introduced.

Definition 4. At each time point $t \in T$ the episodic knowledge base is always represented as a pair:

$$
(\overline{\operatorname{Episodes}(t)}, \underline{\text { Episodes }(t)}),
$$

where:

$\overline{E p i s o d e s(t)}$ states for the experience which is located at the shallow cognitive level (at the time point $t$ ),

Episodes $(t)$ states for the remaining experience (located at the deep cognitive level and at the same $t)$,

Episodes $(t)=\overline{\text { Episodes }(t)} \cup$ Episodes $(t)$,

$\overline{\text { Episodes }(t)} \cap$ Episodes $(t)=\emptyset$.

\section{2. Repository of semantic structures}

As it has already been mentioned above, the episodic memories are summarized into higher level cognitive structures called holons, each reflecting a particular dimension (portion) of knowledge and current state of knowledge elaboration. In our research the state of semantic knowledge base changes along the time line, being related to consecutive states of collected knowledge elaboration. Each dimension (portion) of knowledge is restricted to this object $o$ and experienced appearances of these two properties $P$ and $Q$, which are referred to in particular modal equivalence. In this research we assumed that at each time point the holon assigned to modal equivalence covers both the underlying empirical and relevant material, as well as the related state of elaboration of the material.

Holon is rather uncomplicated, as regards its structure. In particular, in the case of modal equivalence it reflects a mutual relation among four complementary components, each part related to one of four possible distributions of properties $P$ and $Q$ in object $o$. The components can be equated to four normalized numbers, called the relative grounding strengths, reflecting a mutual relation between sizes of sets of episodes called grounding sets, as well as state of grounding sets processing. In case of equivalence-related holon, one component of the holon focuses on the cognitive state relating to the case where both properties $P \in \Omega$ and $Q \in \Omega$ were registered to be exhibited by object $o$. Another component focuses on the cognitive state relating to the case where property $P \in \Omega$ was registered to be exhibited by object $o$ and property $Q \in \Omega$ was registered not to be exhibited by object $o$. The third component focuses on the cognitive state relating to the case where property $P \in \Omega$ was registered not to be exhibited by object $o$ and property $Q \in \Omega$ was registered to be exhibited by object $o$. The final component focuses on the cognitive state relating to the case where both properties $P \in \Omega$ and $Q \in \Omega$, were registered not to be exhibited by object $o$. Below all the formal elements needed to describe the equivalence-related holon will be successively introduced.

Definition 5. At each time point $t \in T$ the following grounding sets related to the logical sense of equivalence are defined, regarding observed appearance of two different properties $P$ and $Q$ in all actual objects of environment:

$$
\begin{array}{r}
C^{p(o) \wedge q(o)}(t)=\left\{E\left(t_{n}\right): t_{n} \leq t, E\left(t_{n}\right) \in \operatorname{Episodes}(t),\right. \\
\left.o \in P^{+}\left(t_{n}\right) \text { and } o \in Q^{+}\left(t_{n}\right)\right\},
\end{array}
$$

$$
\begin{array}{r}
C^{p(o) \wedge \neg q(o)}(t)=\left\{E\left(t_{n}\right): t_{n} \leq t, E\left(t_{n}\right) \in \text { Episodes }(t),\right. \\
\left.o \in P^{+}\left(t_{n}\right) \text { and } o \in Q^{-}\left(t_{n}\right)\right\},
\end{array}
$$

$$
\begin{aligned}
& C^{\neg p(o) \wedge q(o)}(t)=\left\{E\left(t_{n}\right): t_{n} \leq t, E\left(t_{n}\right) \in \text { Episodes }(t),\right. \\
& \left.o \in P^{-}\left(t_{n}\right) \text { and } o \in Q^{+}\left(t_{n}\right)\right\} \text {, }
\end{aligned}
$$

$$
\begin{array}{r}
C^{\neg p(o) \wedge \neg q(o)}(t)=\left\{E\left(t_{n}\right): t_{n} \leq t, E\left(t_{n}\right) \in \operatorname{Episodes}(t),\right. \\
\left.o \in P^{-}\left(t_{n}\right) \text { and } o \in Q^{-}\left(t_{n}\right)\right\} .
\end{array}
$$

Definition 6. At each time point $t \in T$ the grounding sets are represented by the following pairs of processed (shallow) and unprocessed (deep) empirical material, reflecting current state of the episode base processing:

$$
\left(\overline{C^{p(o) \wedge q(o)}(t)}, \underline{\left.C^{p(o) \wedge q(o)}(t)\right)},\right.
$$

where:

$$
\begin{aligned}
\overline{C^{p(o) \wedge q(o)}(t)} & =C^{p(o) \wedge q(o)}(t) \cap \overline{\text { Episodes }(t)} \\
\underline{C^{p(o) \wedge q(o)}(t)} & =C^{p(o) \wedge q(o)}(t) \cap \underline{\text { Episodes }(t)} \\
& \left(\overline{C^{p(o) \wedge \neg q(o)}(t)}, \underline{\left.C^{p(o) \wedge \neg q(o)}(t)\right)},\right.
\end{aligned}
$$

where:

$$
\begin{aligned}
& \overline{C^{p(o) \wedge \neg q(o)}(t)}=C^{p(o) \wedge \neg q(o)}(t) \cap \overline{E p i s o d e s(t)} \\
& \underline{C^{p(o) \wedge \neg q(o)}(t)}=C^{p(o) \wedge \neg q(o)}(t) \cap \underline{\operatorname{Episodes}(t)} \\
& (\overline{C \neg p(o) \wedge q(o)}(t), \underline{C \neg p(o) \wedge q(o)}(t)),
\end{aligned}
$$

where: 
$\overline{C \neg p(o) \wedge q(o)(t)}=C \neg p(o) \wedge q(o)(t) \cap \overline{\text { Episodes }(t)}$

$\underline{C} \neg p(o) \wedge q(o)(t)=C \neg p(o) \wedge q(o)(t) \cap \underline{\operatorname{Episodes}(t)}$

$$
(\overline{C \neg p(o) \wedge \neg q(o)(t)}, \underline{C \neg p(o) \wedge \neg q(o)}(t)),
$$

where:

$\overline{C \neg p(o) \wedge \neg q(o)(t)}=C \neg p(o) \wedge \neg q(o)(t) \cap \overline{\operatorname{Episodes}(t)}$
$C \neg p(o) \wedge \neg q(o)(t)=C \neg p(o) \wedge \neg q(o)(t) \cap \operatorname{Episodes}(t)$

Definition 7. Let the following symbols be given:

$\bar{a}=\left|\overline{C^{p(o) \wedge q(o)}(t)}\right| ; \underline{a}=\mid \underline{C^{p(o) \wedge q(o)}(t) \mid} ; a=\bar{a}+\underline{a}$

$\bar{b}=\left|\overline{C^{p(o) \wedge \neg q(o)}(t)}\right| ; \underline{b}=\left|\underline{C^{p(o) \wedge \neg q(o)}(t)}\right| ; b=\bar{b}+\underline{b}$

$\bar{c}=|\overline{C \neg p(o) \wedge q(o)(t)}| ; \underline{c}=\mid \underline{C \neg p(o) \wedge q(o)(t) \mid} ; c=\bar{c}+\underline{c}$

$\bar{d}=|\overline{C \neg p(o) \wedge \neg q(o)(t)}| ; \underline{d}=|C \neg p(o) \wedge \neg q(o)(t)| ; d=\bar{d}+\underline{d}$

At each time point $t \in T$ the relative grounding strengths, describing the sense of logical equivalence of simultaneous appearance of properties $P$ and $Q$ in object $o$, are given as follows:

$$
\begin{aligned}
& \lambda^{p(o) \Leftrightarrow q(o)}(t)=\frac{\bar{a}+\bar{d}}{a+b+c+d} \\
& \lambda^{p(o) \Leftrightarrow \neg q(o)}(t)=\frac{\bar{b}+\bar{c}}{a+b+c+d} \\
& \lambda \neg p(o) \Leftrightarrow q(o)(t)=\frac{\bar{b}+\bar{c}}{a+b+c+d} \\
& \lambda \neg p(o) \Leftrightarrow \neg q(o)(t)=\frac{\bar{a}+\bar{d}}{a+b+c+d}
\end{aligned}
$$

- simple modalities [6,9],

- modal conjunctions [12, 13, 23],

- modal exclusive and inclusive alternatives [9, 12], and

- modal conditionals $[15,26]$.

\section{3. System of modality thresholds}

The system of modality thresholds is the last important element of the autonomous system architecture, used in the definition of cognitive semantics of modal equivalences listed in tables I and II. The system is responsible for representing the ranges of relative grounding strengths associated with the possibility of using particular modal operators as modality markers in modal equivalences. It was generally assumed in our previous research that with each case of autoepistemic modality (knowledge/necessity, strong belief, weak belief) it is reasonable to assign two dedicated modal thresholds. In this research we verify applicability of the following cases of modality thresholds: $\lambda_{\operatorname{minPos}}^{\Leftrightarrow}$ and $\lambda \dot{\max }_{\operatorname{mos}}$ to restrict the applicability of weak belief marker Pos, $\lambda_{\operatorname{minBel}}^{\Leftrightarrow}$ and $\lambda_{\operatorname{maxBel}}^{\Leftrightarrow}$ to restrict strong belief marker $\mathrm{Bel}$, and a single threshold $\lambda_{K}^{\leftrightarrow_{\text {now }}}$ to capture the case of autoepistemic necessity. Moreover, similarly to other cognitive semantics [6] [12] [9] [26], the following pragmatically justified relationship between the values of introduced thresholds is assumed:

$$
\begin{aligned}
0 & =\lambda_{\operatorname{minPos}}^{\stackrel{\Leftrightarrow}{\Leftrightarrow}}<\lambda_{\operatorname{maxPos}}^{\Leftrightarrow}= \\
& =\lambda_{\operatorname{minBel}}^{\Leftrightarrow}<\lambda_{\operatorname{maxBel}}^{\Leftrightarrow}=\lambda \Leftrightarrow_{\text {now }}=1
\end{aligned}
$$

It is worth mentioning that in each particular natural language determination of the value of thresholds and their mutual relationship is most likely the result of the long-term natural process of semiosis, realized autonomously by population that uses a specific natural language. Such processes are also considered and tested for populations consisting of artificial autonomous systems, e.g. [21] [20].

\section{COGNITIVE SEMANTICS OF MODAL EQUIVALENCES}

The above definitions of concepts and terms associated with the autonomous system architecture considered in this research makes it possible to establish the following detailed definitions of cognitive semantics, each for a particular combination of autoepistemic modality operator and equivalence. For the order of presentation, the definitions are grouped due to generalized protoforms $\Omega(p(o) \Leftrightarrow q(o))$ $\Omega(p(o) \Leftrightarrow \neg q(o)) \Omega(\neg p(o) \Leftrightarrow q(o)) \Omega(\neg p(o) \Leftrightarrow \neg q(o))$, each covering modal equivalence extensions with the same logical sense.

In forthcoming definitions, an original relation of autoepistemic formula satisfaction is introduced, and refered to by dedicated symbol $\models_{G}$. The relation is introduced to specify in technical terms pragmatic conditions for the correct (pragmatically adequate) linking of the current state of knowledge processing with particular linguistic representations, given in the form of modal equivalences. Due to the fact that in this research modal equivalences are studied, the state of knowledge processing is given by content of the above introduced holon, always related to a particular time point $t$. We will further refer to the holon by symbol $P S(t)$.

\section{1. Cognitive semantics for instances of protoform $\Omega(p(o) \Leftrightarrow q(o))$}

According to the content of Table II, there exist three instantiations of the protoform $\Omega(p(o) \Leftrightarrow q(o))$ to be taken 
into account in the current section. In the table instantiations are represented by related modal formulas and additionally described by semi-controlled natural language statements used to communicate commonsense meaning assigned to the formulas. The cognitive semantics is given in the form of the so-called autoepistemic formula satisfaction relation. The relation binds a formula with a list of conditions defining the correct use of the formula by the autonomous system as a surface linguistic representation. The correct use of the formula takes place for those knowledge processing states $P S(t)$ in which all conditions listed in the definition of this formula's cognitive semantics are met. From a practical point of view all definitions formulated below for particular formulas describe the specific internal circumstances that allow the autonomous system to adequately use auto-epistemic modal sentences corresponding to these formulas as an external message describing the current state of knowledge.

Definition 8. The relation of autoepistemic satisfaction of the formula $S P(t) \models_{G} \operatorname{Pos}(p(o) \Leftrightarrow q(o))$, representing cognitive semantics of modal equivalence $\operatorname{Pos}(p(o) \Leftrightarrow$ $q(o))$, holds if and only if:

$$
\begin{gathered}
\left(\overline{C^{p(o) \wedge \neg q(o)}(t)} \cup \overline{C \neg p(o) \wedge q(o)(t)}=\emptyset\right. \\
\wedge \\
\left.\overline{C^{p(o) \wedge q(o)}(t)}=\emptyset\right) \\
\left(\frac{o r}{C^{p(o) \wedge \neg q(o)}(t)} \cup \overline{C \neg p(o) \wedge q(o)(t)}=\emptyset\right. \\
\wedge \\
\left.\lambda_{\operatorname{minPos}} \leq \lambda^{p(o) \Leftrightarrow q(o)}(t)<\lambda_{\operatorname{maxPos}}^{\Leftrightarrow}\right)
\end{gathered}
$$

Definition 9. The relation of autoepistemic satisfaction of the formula $P S(t) \models_{G} \operatorname{Bel}(p(o) \Leftrightarrow q(o))$, representing cognitive semantics of modal equivalence $\operatorname{Bel}(p(o) \Leftrightarrow$ $q(o))$, holds if and only if:

$$
\begin{gathered}
\overline{C^{p(o) \wedge \neg q(o)}(t)} \cup \overline{C^{\neg p(o) \wedge q(o)}(t)}=\emptyset \\
\lambda_{\operatorname{minBel}}^{\Leftrightarrow} \leq \lambda^{p(o) \Leftrightarrow q(o)}(t)<\lambda_{\operatorname{maxBel}}^{\Leftrightarrow} \\
\overline{C^{p(o) \wedge q(o)}(t)} \neq \emptyset
\end{gathered}
$$

Definition 10. The relation of autoepistemic satisfaction of the formula $P S(t) \models_{G} \operatorname{Know}(p(o) \Leftrightarrow q(o))$, representing cognitive semantics of modal equivalence $\operatorname{Know}(p(o) \Leftrightarrow$ $q(o))$, holds if and only if:

$$
\begin{aligned}
& \underline{C^{p(o) \wedge q(o)}(t)} \cup \underline{C^{p(o) \wedge \neg q(o)}(t)} \cup \\
& \underline{\cup C \neg p(o) \wedge q(o)}(t) \cup \underline{C \neg p(o) \wedge \neg q(o)}(t)=\emptyset \\
& \overline{C^{p(o) \wedge \neg q(o)}(t)} \cup \overline{C \neg p(o) \wedge q(o)(t)}=\emptyset \\
& \lambda_{K}^{\Leftrightarrow} \stackrel{\leftrightarrow}{n o w} \leq \lambda^{p(o) \Leftrightarrow q(o)}(t)=1 \\
& \overline{C^{p(o) \wedge q(o)}(t)}=C^{p(o) \wedge q(o)}(t) \neq \emptyset
\end{aligned}
$$

In the analogous way below, we define and comment on the pragmatic function of cognitive semantics defined for all instances of the other protoforms of modal equivalence.

\section{2. Cognitive semantics for instances of protoform $\Omega(p(o) \Leftrightarrow \neg q(o))$}

Definition 11. The relation of autoepistemic satisfaction of the formula $\operatorname{PS}(t) \models_{G} \operatorname{Pos}(p(o) \Leftrightarrow \neg q(o))$, representing cognitive semantics of modal equivalence $\operatorname{Pos}(p(o) \Leftrightarrow$ $\neg q(o))$, holds if and only if:

$$
(\overline{C p(o) \wedge \neg q(o)}(t) \cup \overline{C \neg p(o) \wedge q(o)(t)}=\emptyset
$$

$$
\begin{aligned}
& \wedge \\
& \left.\overline{C^{p(o) \wedge \neg q(o)}(t)}=\emptyset\right) \\
& \text { or } \\
& (\overline{C p(o) \wedge \neg q(o)(t)} \cup \overline{C \neg p(o) \wedge q(o)(t)}=\emptyset \\
& \wedge \\
& \left.\lambda_{\operatorname{minPos}}^{\Leftrightarrow} \leq \lambda^{p(o) \Leftrightarrow \neg q(o)}(t)<\lambda_{\operatorname{maxPos}}^{\Leftrightarrow}\right)
\end{aligned}
$$

Definition 12. The relation of autoepistemic satisfaction of the formula $P S(t) \models_{G} \operatorname{Bel}(p(o) \Leftrightarrow \neg q(o))$, representing cognitive semantics of modal equivalence $\operatorname{Bel}(p(o) \Leftrightarrow$ $\neg q(o))$, holds if and only if:

$$
\begin{gathered}
\overline{C p(o) \wedge q(o)(t)} \cup \overline{C \neg p(o) \wedge \neg q(o)(t)}=\emptyset \\
\lambda_{m i n B e l}^{\Leftrightarrow} \leq \lambda^{p(o) \Leftrightarrow \neg q(o)}(t)<\lambda_{\max B e l}^{\Leftrightarrow} \\
\overline{C^{p(o) \wedge \neg q(o)}(t)} \neq \emptyset
\end{gathered}
$$


Definition 13. The relation of autoepistemic satisfaction of the formula $P S(t) \models_{G} \quad \operatorname{Know}(p(o) \Leftrightarrow \neg q(o))$, representing cognitive semantics of modal equivalence $\operatorname{Know}(p(o) \Leftrightarrow \neg q(o))$, holds if and only if:

$$
\begin{aligned}
& \underline{C^{p(o) \wedge q(o)}(t)} \cup \underline{C^{p(o) \wedge \neg q(o)}(t) \cup} \\
& \underline{\cup C \neg p(o) \wedge q(o)}(t) \cup \underline{C \neg p(o) \wedge \neg q(o)}(t)=\emptyset \\
& \overline{C^{p(o) \wedge q(o)}(t)} \cup \overline{C \neg p(o) \wedge \neg q(o)(t)}=\emptyset \\
& \lambda_{K}^{\Leftrightarrow}{ }_{\text {now }} \leq \lambda^{p(o) \Leftrightarrow \neg q(o)}(t)=1 \\
& \overline{C^{p(o) \wedge \neg q(o)}(t)}=C^{p(o) \wedge \neg q(o)}(t) \neq \emptyset
\end{aligned}
$$

\section{3. Cognitive semantics for instances of protoform $\Omega(\neg p(o) \Leftrightarrow q(o))$}

Definition 14. The relation of autoepistemic satisfaction of the formula $P S(t) \models_{G} \operatorname{Pos}(\neg p(o) \Leftrightarrow q(o))$, representing cognitive semantics of modal equivalence $\operatorname{Pos}(\neg p(o) \Leftrightarrow$ $q(o))$, holds if and only if:

$$
\begin{aligned}
& \left(\overline{C^{p(o) \wedge q(o)}(t)} \cup \overline{C \neg p(o) \wedge \neg q(o)}(t)=\emptyset\right. \\
& \wedge \\
& \overline{C \neg p(o) \wedge q(o)(t)}=\emptyset) \\
& \text { or }
\end{aligned}
$$

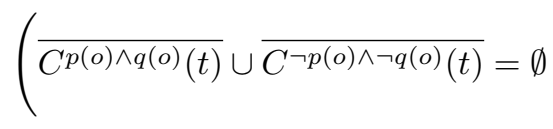

$$
\begin{aligned}
& \wedge \\
& \left.\lambda_{\operatorname{minPos}}^{\Leftrightarrow} \leq \lambda^{\neg p(o) \Leftrightarrow q(o)}(t)<\lambda_{\operatorname{maxPos}}^{\Leftrightarrow}\right)
\end{aligned}
$$

Definition 15. The relation of autoepistemic satisfaction of the formula $P S(t) \models_{G} \operatorname{Bel}(\neg p(o) \Leftrightarrow q(o))$, representing cognitive semantics of modal equivalence $\operatorname{Bel}(\neg p(o) \Leftrightarrow$ $q(o))$, holds if and only if:

$$
\begin{gathered}
\overline{C^{p(o) \wedge q(o)}(t)} \cup \overline{C \neg p(o) \wedge \neg q(o)(t)}=\emptyset \\
\lambda_{\operatorname{minBel}}^{\Leftrightarrow} \leq \lambda^{\neg p(o) \Leftrightarrow q(o)}(t)<\lambda_{\max B e l}^{\Leftrightarrow} \\
\overline{C^{\neg p(o) \wedge q(o)(t)}} \neq \emptyset
\end{gathered}
$$

Definition 16. The relation of autoepistemic satisfaction of the formula PS(t) $\models_{G} \quad \operatorname{Know}(\neg p(o) \Leftrightarrow q(o))$, representing cognitive semantics of modal equivalence Know $(\neg p(o) \Leftrightarrow q(o))$, holds if and only if:

$$
\begin{aligned}
& \underline{C^{p(o) \wedge q(o)}(t)} \cup \underline{C^{p(o) \wedge \neg q(o)}(t) \cup} \\
& \underline{\cup C \neg p(o) \wedge q(o)}(t) \cup \underline{C \neg p(o) \wedge \neg q(o)}(t)=\emptyset \\
& \overline{C^{p(o) \wedge q(o)}(t)} \cup \overline{C^{\neg p(o) \wedge \neg q(o)}(t)}=\emptyset \\
& \lambda_{K}^{\stackrel{\Leftrightarrow}{K} \text { now }} \leq \lambda^{\neg p(o) \Leftrightarrow q(o)}(t)=1 \\
& \overline{C \neg p(o) \wedge q(o)(t)}=C \neg p(o) \wedge q(o)(t) \neq \emptyset
\end{aligned}
$$

\section{4. Cognitive semantics for instances of protoform} $\Omega(\neg p(o) \Leftrightarrow \neg q(o))$

Definition 17. The relation of autoepistemic satisfaction of the formula $P S(t) \models_{G} \operatorname{Pos}(\neg p(o) \Leftrightarrow \neg q(o))$, representing cognitive semantics of modal equivalence $\operatorname{Pos}(\neg p(o) \Leftrightarrow$ $\neg q(o))$, holds if and only if:

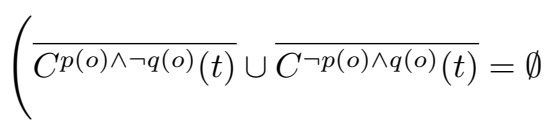

$$
\begin{gathered}
\wedge \\
\overline{C \neg p(o) \wedge \neg q(o)(t)}=\emptyset) \\
o r \\
(\overline{C p(o) \wedge \neg q(o)(t)} \cup \overline{C \neg p(o) \wedge q(o)(t)}=\emptyset \\
\wedge \\
\left.\lambda_{\operatorname{minPos}} \leq \lambda \neg p(o) \Leftrightarrow \neg q(o)(t)<\lambda \Leftrightarrow \operatorname{maxPos}\right)
\end{gathered}
$$

Definition 18. The relation of autoepistemic satisfaction of the formula $P S(t) \models_{G} \operatorname{Bel}(\neg p(o) \Leftrightarrow \neg q(o))$, representing cognitive semantics of modal equivalence $\operatorname{Bel}(\neg p(o) \Leftrightarrow$ $\neg q(o))$, holds if and only if:

$$
\begin{gathered}
\overline{C p(o) \wedge \neg q(o)(t)} \cup \overline{C \neg p(o) \wedge q(o)(t)}=\emptyset \\
\lambda_{m i n B e l}^{\Leftrightarrow} \leq \lambda \neg p(o) \Leftrightarrow \neg q(o)(t)<\lambda_{\max B e l}^{\Leftrightarrow} \\
\overline{C \neg p(o) \wedge \neg q(o)(t)} \neq \emptyset
\end{gathered}
$$


Definition 19. The relation of autoepistemic satisfaction of the formula $P S(t) \models_{G} \quad K n o w(\neg p(o) \Leftrightarrow \neg q(o))$, representing cognitive semantics of modal equivalence Know $(\neg p(o) \Leftrightarrow \neg q(o))$, holds if and only if:

$$
\begin{aligned}
& \underline{\cup \frac{C^{p(o) \wedge q(o)}(t)}{U} \cup \underline{C^{\neg p(o) \wedge q(o)}(t)} \cup \underline{C^{\neg p(o) \wedge \neg q(o)}(t)}=\emptyset} \\
& \overline{C^{p(o) \wedge \neg q(o)}(t)} \cup \overline{C^{\neg p(o) \wedge q(o)}(t)}=\emptyset
\end{aligned}
$$

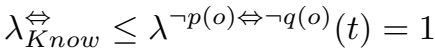

$$
\begin{aligned}
& \overline{C \neg p(o) \wedge \neg q(o)}(t)=C\urcorner p(o) \wedge \neg q(o)(t) \neq \emptyset
\end{aligned}
$$

\section{NONEMPTINESS OF THE DEFINITIONS FOR COGNITIVE SEMANTICS}

Our analysis of properties of the modal equivalence cognitive semantics begins with the proof of theorems that state that the proposed definitions are not "empty" in the sense that each case of semantics has at least one possible state of empirical knowledge processing, encapsulated in the considered autonomous system. The formulated property and its verification constitute the main subject of the analysis carried out in this article. The analysis of other important properties of the cognitive semantics, including the coincidence of the artificial language behavior based on the semantics with the natural language behavior, is beyond the scope of this paper.

In order to demonstrate the non-emptiness of the proposed cognitive semantics for each case of the modal equivalence, possible cognitive states of the autonomous system will be explicitly constructed, understood as the states of processing of collection of collected episodes, meeting the conditions of cognitive semantics definition related to a given case of equivalence. To justify the non-emptiness of definitions in its essence will be based on the analysis of behaviour of relatively simple functions of one or two variables.

We will start the proof with modal equivalences being surface linguistic representations of the logical sense attributed to higher level protoform $\Pi(p(o) \Leftrightarrow q(o))$, where $\Pi \in\{P o s, B e l, K$ now $\}$. The related theorem is as follows:

Theorem 1. In the autonomous system of knowledge that uses the relation of autoepistemic satisfaction of modal equivalence to choose linguistic representations of its knowledge, it is possible to determine at least one state of processing of empirical knowledge base PS(t) that fulfills the definitional conditions of cognitive semantics $P S(t) \models \models_{G}$ $\Pi(p(o) \Leftrightarrow q(o))$. The autonomous system possesses the characteristic for every autoepistemic operator $\Pi \in$ $\{$ Know, Bel, Pos\}.

Proof. To simplify the presentation of proof, let us enter the following auxiliary symbols:

$\bar{a}=\left|\overline{C^{p(o) \wedge q(o)}(t)}\right| ; \underline{a}=\left|\underline{C^{p(o) \wedge q(o)}(t)}\right| ; a=\bar{a}+\underline{a}$

$\bar{b}=\left|\overline{C^{p(o) \wedge \neg q(o)}(t)}\right| ; \underline{b}=\left|C^{p(o) \wedge \neg q(o)}(t)\right| ; b=\bar{b}+\underline{b}$

$\bar{c}=|\overline{C \neg p(o) \wedge q(o)(t)}| ; \underline{c}=\left|C^{\neg p(o) \wedge q(o)}(t)\right| ; c=\bar{c}+\underline{c}$

$\bar{d}=|\overline{C \neg p(o) \wedge \neg q(o)}(t)| ; \underline{d}=|\underline{C \neg p(o) \wedge \neg q(o)}(t)| ; d=\bar{d}+\underline{d}$

$k=\bar{a}+\bar{d}$

$m=\underline{a}+b+c+\underline{d}$

Verification of the possibility of establishing states of empirical knowledge processing, for short called mental states and fulfilling the considered relation of the autoepistemic satisfaction of the modal equivalence, will be further given separately for each modal operator $\Pi \in$ $\{$ Know, Bel, Pos $\}$. Verification will consist in indicating pairs of natural numbers $\left(k^{*}, m^{*}\right)$, such that after substitution $k:=k^{*}$ i $m:=m^{*}$ a considered case of satisfaction relation of modal equivalence holds.

Determining the numbers $k^{*}$ and $m^{*}$, satisfying the conditions of modal equivalence satisfaction relation, will always be carried out taking into account one of the three additional restrictions imposed on their value, resulting from an additional assumption of non-emptiness of the empirical knowledge base collected by the autonomous system. Using the auxiliary symbols introduced above, we specify the assumption about non-emptiness of the empirical knowledge database by the following limiting condition:

$$
k+m \in \mathbb{N} \backslash\{0\} .
$$

Quite obviously, meeting the constraint 54 results in reducing the possible range of natural numbers $k^{*}$ and $m^{*}$ to the following three specific cases:

$\left(1^{\circ}\right) k^{*} \geq 1, m^{*}=0$

$\left(2^{\circ}\right) k^{*}=0, m^{*} \geq 1$

$\left(3^{\circ}\right) k^{*} \geq 1, m^{*} \geq 1$

Proving the existence of states fulfilling the autoepistemic satisfaction relation for equivalence $\operatorname{Pos}(p(o) \Leftrightarrow q(o))$ :

Possibility of entering the autonomous system into a state of empirical knowledge processing described with modal equivalence $\operatorname{Pos}(p(o) \Leftrightarrow q(o))$ is proved for each case $\left(1^{\circ}\right)$ - $\left(3^{\circ}\right)$ by indicating, if it is possible, specific natural numbers $k$ and $m$, meeting at the same time the minimum and maximum definitional conditions:

$$
0=\lambda_{M}^{\stackrel{\leftrightarrow}{i n P o s}} \leq \frac{k}{k+m}
$$




$$
\frac{k}{k+m}<\lambda_{M}^{\leftrightarrow} \text { axPos }
$$

A set of potential solutions, limited to $\left(1^{\circ}\right)$, does not consist of any solution which would meet conditions 55 and 56. Indeed, for $m=0$ we have:

$$
\begin{aligned}
& \lambda^{p(o) \Leftrightarrow q(o)}(t)=\frac{k}{k+0}=1> \\
& >\lambda_{\stackrel{M}{a x P o s}}>\lambda_{\stackrel{M}{M}_{\text {inPos }}}=0 .
\end{aligned}
$$

The given property is fully consistent with the desirable artificial pragmatic (commonsense) interpretation of the auto-epistemic satisfaction relation, because the establishment of any state of knowledge, meeting the limitations of $\left(1^{\circ}\right)$, is equivalent to full processing of empirical knowledge, and thus entering of the autonomous system into the state of autoepistemic certainty. Such a state of autoepistemic certainty excludes the legitimacy and need for the autonomous system to use the operator Pos at the level of adequate surface linguistic representations.

In turn, the constraints of case $\left(2^{\circ}\right)$ allow to specify an infinite number of concretization of states of empirical knowledge processing that meet the definitional conditions 55 and 56. For this situation, we obtain the following relationship between the value of the relative grounding strength, for the considered case of modal equivalence, and the modality thresholds:

$$
\begin{aligned}
& \lambda_{\stackrel{\leftrightarrow}{M}{ }_{a x P o s}>\lambda^{p(o) \Leftrightarrow q(o)}(t)=} \\
& =\frac{0}{0+m}=0 \geq \lambda_{\stackrel{M}{M} \text { inPos }} .
\end{aligned}
$$

Thus, both definitional conditions 55 and 56 are fulfilled. It is worth noting, however, that the considered class of concretization of empirical knowledge processing states corresponds to a very specific and non-trivial cognitive state. This is a state of complete lack of awareness of the content of empirical knowledge available to the autonomous system, regarding the logical meaning of protoform $\operatorname{Pos}(p(o) \Leftrightarrow$ $q(o))$. It is a state cognitively opposite to the situation described by the case $\left(1^{\circ}\right)$, and all acceptable solutions $\left(k^{*}, m^{*}\right)$ take the form:

$$
\begin{array}{r}
k^{*}=0, \\
m^{*} \in \mathbb{N} \backslash\{0\} .
\end{array}
$$

Clearly, the set of acceptable solutions becomes infinite. The set of acceptable solutions $\left(k^{*}, m^{*}\right)$ limited by conditions of the case $\left(3^{\circ}\right)$ it is not empty, either.

First of all, let us show that in any autonomous system, using the accepted system of modality thresholds, it is possible to indicate some states of empirical knowledge processing that fulfill condition 56 in the definition of cognitive semantics of linguistic instantiations of protoform $\operatorname{Pos}(p(o) \Leftrightarrow q(o))$. To do this, let us make the following transformations:

$$
\begin{aligned}
& \lambda^{\Leftrightarrow}(t)=\frac{k}{k+m}<\lambda_{M}^{\leftrightarrow} \text { ax Pos }, \\
& k<k \cdot \lambda_{\stackrel{M}{a x P o s}}+m \cdot \lambda_{M}^{\Leftrightarrow} \text { axPos }
\end{aligned}
$$

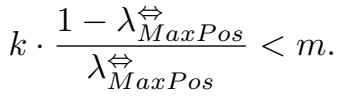

In a simple way, we can now show that for every number $k^{*} \in \mathbb{N} \backslash\{0\}$ there exist infinitely many numbers $m^{*}$ such that the pair $\left(k^{*}, m^{*}\right)$ meets the upper definitional requirement 56. Indeed, from the following property of the modality threshold system:

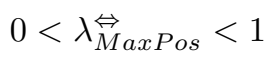

it follows that:

$$
0<\frac{1-\lambda_{M}^{\leftrightarrow} a x P o s}{\lambda_{M}^{\leftrightarrow} a x P o s},
$$

which after considering the condition $k \geq 1$, adopted for the case $3^{\circ}$, leads to the conclusion:

$$
0<k \cdot \frac{\left(1-\lambda_{\stackrel{\leftrightarrow}{M} a x P o s}\right)}{\lambda_{M}^{\leftrightarrow} a x P o s},
$$

and next, for all $i \in \mathbb{N} \backslash\{0\}$, to the conclusion:

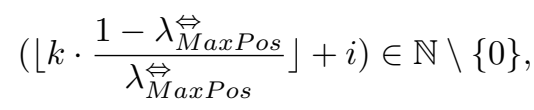

where $\lfloor x\rfloor$ denotes the floor of number $x$.

From the above it follows that to indicate a specific state of empirical knowledge processing, fulfilling the upper definitional condition 56, it is enough to consider any three natural numbers $k^{*}, m^{*}$ and $i$ fulfilling the conditions:

$$
\begin{array}{r}
k^{*} \in \mathbb{N} \backslash\{0\}, \\
i \in \mathbb{N} \backslash\{0\}, \\
m^{*}=\left\lfloor k \cdot \frac{1-\lambda_{\dot{M} a x P o s}^{\Leftrightarrow}}{\lambda_{\dot{M}}^{\leftrightarrow} \text { axPos }}\right\rfloor+i,
\end{array}
$$

and then make substitutions $k:=k^{*}$ and $m:=m^{*}$.

In the next step, it should be noted that values $k^{*}$ and $m^{*}$ determined assuming that conditions 65 are taken into account, fulfill the lower definitional condition (55) in the definition of modal equivalence cognitive semantics, representing the logical sense of protoform $\operatorname{Pos}(p(o) \Leftrightarrow q(o))$. Indeed, for every two positive numbers, and therefore also 
for numbers $k:=k^{*}$ and $m:=m^{*}$ determined as above, it obviously occurs that:

$$
\lambda^{p(o) \Leftrightarrow q(o)}(t)=\frac{k}{k+m} \geq \lambda_{M \text { inPos }}=0 .
$$

Also for the case $\left(3^{\circ}\right)$ we have pointed out that there exists an infinite set of states of empirical knowledge processing that fulfill definitional conditions of cognitive semantics of modal equivalence, linguistically representing the meaning of protoform $\operatorname{Pos}(p(o) \Leftrightarrow q(o))$.

In consequence, the above analysis shows that for the protoform $\operatorname{Pos}(p(o) \Leftrightarrow q(o))$ the proposed definition of cognitive semantics of the modal equivalences is not empty.

Proving the existence of states fulfilling the autoepistemic satisfaction relation for equivalence $\operatorname{Bel}(p(o) \Leftrightarrow q(o))$.

Similarly to the case of operator Pos, the possibility of entering the autonomous system into the knowledge processing state described by the modal equivalence $\operatorname{Bel}(p(o) \Leftrightarrow$ $q(o))$ is shown, if it is possible, for each case $\left(1^{\circ}\right)-\left(3^{\circ}\right)$ by determining specific values of natural numbers $k$ and $m$, simultaneously meeting the minimum and maximum definitional conditions:

$$
\begin{gathered}
\lambda_{M}^{\stackrel{\leftrightarrow}{\text { inBel }}} \leq \frac{k}{k+m} \\
\frac{k}{k+m}<1=\lambda_{\stackrel{\leftrightarrow}{M} a x B e l}
\end{gathered}
$$

A set of acceptable solutions belonging to the area designated by the condition $\left(1^{\circ}\right)$ it is empty, as in the case of protoform $\operatorname{Pos}(p(o) \Leftrightarrow q(o))$. Namely, the value of relative grounding strength $\lambda^{p(o) \Leftrightarrow q(o)}(t)=\frac{k}{k+m}=1$. In consequence, conditions 67 and 68 are not fulfilled. A comment on the commonsense consistency of the just described behavior with the pragmatics of natural language production is analogous to our previous comment related to the protoform $\operatorname{Pos}(p(o) \Leftrightarrow q(o))$.

A set of acceptable solutions that meet the conditions of the event $\left(2^{\circ}\right)$ is empty, either. It follows directly from the fact that the following relationships between the relative grounding strength and modality thresholds accepted for operator Bel occur:

$$
\begin{aligned}
& \lambda^{p(o) \Leftrightarrow q(o)}(t)=\frac{0}{0+m}=
\end{aligned}
$$

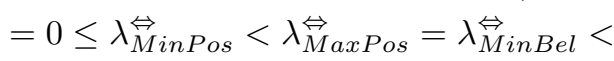

$$
\begin{aligned}
& <\lambda_{\stackrel{M}{a x B e l}}=1 \text {. }
\end{aligned}
$$

In situations described by equation 69 , none of the definitional conditions 67 and 68 is satisfied.
However, to determine the solutions of inequalities forming the content of both definitional conditions 67 and 68, fulfilling additional restriction given by case $\left(3^{\circ}\right)$, we follow a procedure as for case $\left(3^{\circ}\right)$, provided that another protoform $\operatorname{Pos}(p(o) \Leftrightarrow q(o))$ is taken into account.

In the first step, we show the existence of acceptable solutions for the definitional condition 67. To achieve such goal, we realize the following transformations:

$$
\begin{aligned}
& \lambda_{M \text { inBel }} \leq \frac{k}{k+m}=\lambda^{\Leftrightarrow}(t), \\
& \lambda_{\stackrel{M}{\text { inBel }}_{\text {in }}} \cdot(k+m) \leq k, \\
& m \cdot \frac{\lambda \stackrel{\leftrightarrow}{M i n B e l}_{1-\lambda \stackrel{\leftrightarrow}{M i n B e l}_{i}}}{1-} k .
\end{aligned}
$$

Then we show in a simple way that for every number $m^{*} \in$ $\mathbb{N} \backslash\{0\}$ there exists an infinite set of numbers $k^{*}$ such that each pair $\left(k^{*}, m^{*}\right)$ fulfills the definitional condition 67. Referring to the following property of the modality threshold system we have:

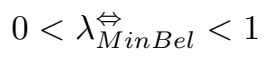

and conclude:

$$
0<\frac{\lambda_{M}^{\stackrel{\leftrightarrow}{i n B e l}}}{1-\lambda_{M}^{\stackrel{\leftrightarrow}{M} B e l}} .
$$

Then, after taking the condition $m \geq 1$ into account, accepted for case $3^{\circ}$, we have:

$$
0<m \cdot \frac{\lambda \stackrel{\leftrightarrow}{\stackrel{\leftrightarrow}{i n B e l}}}{1-\lambda \stackrel{\leftrightarrow}{M} i n B e l_{i n}},
$$

and in consequence, for each $i \in \mathbb{N} \backslash\{0\}$, we have:

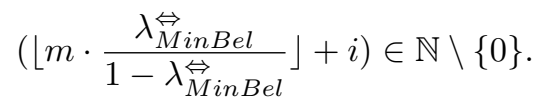

Similarly to the autoepistemic operator of possibility, it follows from the above that in order to indicate a specific state of empirical knowledge processing that fulfills the definitional condition 67 , it is enough to consider any three numbers $k^{*}, m^{*}$ and $i$ fulfilling the following requirements:

$$
\begin{array}{r}
m^{*} \in \mathbb{N} \backslash\{0\}, \\
i \in \mathbb{N} \backslash\{0\}, \\
k^{*}=\left\lfloor m \cdot \frac{\lambda \stackrel{\leftrightarrow}{M} \text { inBel }}{1-\lambda_{M}^{\leftrightarrow} \text { inBel }}\right\rfloor+i,
\end{array}
$$

and then carry out the substitutions $k:=k^{*}$ i $m:=m^{*}$.

It should also be noted that the values $k^{*}$ and $m^{*}$, determined with respect to requirements 75 , fulfill the definitional 
condition 68 proposed for using modal equivalences representing logical sens of the protoform $\operatorname{Bel}(p(o) \Leftrightarrow q(o))$ as surface linguistic representations of internal knowledge states. There is:

$$
\lambda^{p(o) \Leftrightarrow q(o)}(t)=\frac{k}{k+m}<1=\lambda_{M}^{\Leftrightarrow} a x B e l .
$$

The above concludes the proof that it is possible for the autonomous system to enter such states of empirical knowledge processing (called mental states) that require the use of linguistic representations of the logical sense covered with protoform $\operatorname{Bel}(p(o) \Leftrightarrow q(o))$.

Proving the existence of states fulfilling the autoepistemic satisfaction relation for equivalence Know $(p(o) \Leftrightarrow q(o))$. To prove the possibility of entering the autonomous system into a state of knowledge requiring the use of modal equivalence $\operatorname{Know}(p(o) \Leftrightarrow q(o))$ it is enough to notice that each pair of possible solutions $(k, m)$, taking into account restrictions defined by case $\left(3^{\circ}\right)$, fulfills obligatory for this protoform:

$$
\lambda_{\stackrel{K}{n o w}_{n o w}}=1 .
$$

Indeed, as it was mentioned for case $\left(3^{\circ}\right)$ the value of relative grounding strength for the case of all linguistically realized modal equivalences representing the logical sense of protoform Know $(p(o) \Leftrightarrow q(o))$ meets the definitional equation $\lambda^{p(o) \Leftrightarrow q(o)}(t)=\frac{k}{k+0}=1$. Thus, after the fulfillment of additional definitional conditions imposed on the sets of empirical data $\overline{C^{p(o) \wedge q(o)}(t)} \overline{C \neg p(o) \wedge \neg q(o)}(t)$, consisting of episodes related to cases of modal equivalences being considered, it is possible to determine specific states of knowledge processing $P S(t)$ requiring reference to protoform $\operatorname{Know}(p(o) \Leftrightarrow q(o))$.

In the summary, we conclude that the proposed relation of autoepistemic satisfaction of modal equivalence by internal states of empirical knowledge processing, also called mental states of the autonomous agent, for each case of autoepistemic operators of modality modal $\Pi \in$ $\{K$ now, Bel, Pos $\}$ is not empty. It concludes the proof

For the completeness of the presentation of the proposed theory of priming modal equivalences, it should be noted that this theory also includes below three theorems, logically complementary to the theorem 1, i.e. concerning surface linguistic representations of the logical sense of protoforms $\Pi(p(o) \Leftrightarrow \neg q(o)), \Pi(\neg p(o) \Leftrightarrow q(o))$ and $\Pi(\neg p(o) \Leftrightarrow$ $\neg q(o))$, respectively:

Theorem 2. In the autonomous system of knowledge that uses the relation of autoepistemic satisfaction of modal equivalence to choose linguistic representations of its knowledge, it is possible to determine at least one state of processing of empirical knowledge base $P S(t)$ that fulfills the definitional conditions of cognitive semantics $P S(t) \models_{G} \Pi(p(o) \Leftrightarrow \neg q(o))$. The autonomous system posses the characteristic for every autoepistemic operator $\Pi \in\{$ Know, Bel, Pos $\}$.

Proof. The proof is analogous to the proof of theorem 1.

Theorem 3. In the autonomous system of knowledge that uses the relation of autoepistemic satisfaction of modal equivalence to choose linguistic representations of its knowledge, it is possible to determine at least one state of processing of empirical knowledge base PS(t) that fulfills the definitional conditions of cognitive semantics $P S(t) \models_{G}$ $\Pi(\neg p(o) \Leftrightarrow q(o))$. The autonomous system possesses the characteristic for every autoepistemic operator $\Pi \in$ $\{$ Know, Bel, Pos $\}$.

Proof. The proof is analogous to the proof of theorem 1.

Theorem 4. In the autonomous system of knowledge that uses the relation of autoepistemic satisfaction of modal equivalence to choose linguistic representations of its knowledge, it is possible to determine at least one state of processing of empirical knowledge base PS(t) that fulfills the definitional conditions of cognitive semantics $P S(t) \models_{G}$ $\Pi(\neg p(o) \Leftrightarrow \neg q(o))$. The autonomous system possesses the characteristic for every autoepistemic operator $\Pi \in$ $\{$ Know, Bel, Pos $\}$.

Proof. The proof is analogous to the proof of theorem 1.

\section{CONCLUSIONS}

The most important conclusion from the so-called theory of grounding of modal equivalences, the elements of which are the above listed and analyzed theorems on non-emptiness of the cognitive semantics definition, is of pragmatic nature. Namely, while constructing individual theorems of the theory, we prove in an analytical way the following thesis: The semi-controlled language behaviour of the autonomous system covered by the proposed theory of grounding surface linguistic statements in an internal empirical knowledge base and realized including the proposed definitions of cognitive semantics makes it possible to design and implement an artificial cognitive systems capable of artificial linguistic behaviour convergent with natural language behaviour. In this paper such a thesis was partially studied and justified for the case of surface linguistic representations taking the form of modal equivalences.

The reported research is part of a broader and long-term $R \& D$ project within which other classes of modal linguistic representations are studied and utilized, being purely subjective statements of knowledge states of artificial cognitive systems. 


\section{Acknowledgement}

This research was carried out at Wrocław University of Science and Technology (Poland) under Grant 0401/0190/18 titled Models and Methods of Semantic Communication in Cyber-Physical Systems.

\section{References}

[1] P.R. Cohen, H.J. Levesque, Intention is choice with commitment., Artificial Intelligence 42, No. 2-3, pp. 213-269 (1990).

[2] S. Coradeschi, A. Saffiotti, Anchoring symbolic object descriptions to sensory data. Problem statement., Linköping Electronic Articles in Computer and Information Science 4(9) (1999).

[3] J.Y. Halpern, Y. Moses, Knowledge and common knowledge in a distributed environment, Journal of ACM 37, No. 3, pp. 549-587 (1990).

[4] J.Y. Halpern, Y. Moses, A guide to completeness and complexity for modal logics of knowledge and belief., Artificial Intelligence 54, No. 3, pp. 319-379 (1992).

[5] J. Hintikka, Knowledge and belief. An introduction to the logic of the two no-tions., Cornell University Press, New York, 1962.

[6] R.P. Katarzyniak, On some properties of grounding simple modalities, Systems Science 31(3), pp. 59-86 (2005).

[7] R.P. Katarzyniak, Some notes on grounding singletons of modal conjunctions, Systems Science 32(1), pp. 45-55 (2005).

[8] R.P. Katarzyniak, On some properties of grounding uniform sets of modal conjunctions. Journal of Intelligent Fuzzy Systems 17, No. 3, pp. 209-218 (2006).

[9] R.P. Katarzyniak, Gruntowanie modalnego jezyka komunikacji $w$ systemach agentowych, Akademicka Oficyna Wydawnicza Exit, Warszawa, 2007 (in Polish).

[10] R.P. Katarzyniak, W. Lorkiewicz, Więcek D., Some Notes on Extracting Linguistic Summaries Built with Epistemic Modalities and Natural Language Connectives of Equivalence, Computational Methods in Science and Technology 23(1), pp. 19-41 (2017).

[11] R.P. Katarzyniak, N.T. Nguyen and J.C. Jain, Soft computing approach to contextual determinantion of grounding sets for simple modalities, Lecture Notes in Computer Science (Lecture Notes in Artificial Intelligence) 4692, pp. 230-237 (2007).

[12] R.P. Katarzyniak, Pieczyńska-A. Kuchtiak, A consensus based algorithm for grounding belief formulas in internally stored perceptions, Neural Network World 12(5), pp. 461472 (2002).

[13] R.P. Katarzyniak, Pieczyńska-A. Kuchtiak, Grounding and extracting modal re-sponses in cognitive agents: 'and' query and states of incomplete knowledge, International Journal of Applied Mathematics and Computer Science 14(2), pp. 249263 (2004).

[14] S. Kripke, Semantical Analysis of Modal Logic, Zeitschrift für Mathematische Logic und Grundlagen der Mathematik 9, pp. 67-96 (1963).
[15] R.P. Katarzyniak, Więcek D., An approach to extraction of linguistic recommendation rules - application of modal conditionals grounding, Lecture Notes in Computer Science Lecture Notes in Artificial Intelligence 7653, pp. 249-258 (2012).

[16] R.W. Langacker, Foundations of cognitive grammar 1, Theoretical prerequisits, Stanford University Press, Stanford, 1987.

[17] R.W. Langacker, Foundations of cognitive grammar 2, Descrptive application, Stanford University Press, Stanford, 1991.

[18] B. Linder van, W. Hoek van der, J. Meyer-J. Ch., Formalising motivational at-titudes of agents: on preferences, goals, and commitements., [In:] Intelligent Agents II. Agent Theories, architectures, and Languages., M. Wooldridge, Müller J.P., M. Tambe (eds.), Proc. IJCAI'95 (ATAL 1995), pp. 17-32, 1995.

[19] B. Lindern van, W. van der Hoek, J.-J. CH. Meyer, Formalising abilities and opportunities of Agents., Fundamenta Informatica 34, No. 1-2, pp. 53-101 (1998).

[20] W. Lorkiewicz, Issues on Two-Layer Object Perception in the Process of Artificial Semiosis, Third European Network Intelligence Conference (ENIC), Wroclaw, pp. 165-172, 2016.

[21] W. Lorkiewicz, R.P. Katarzyniak, R. Kowalczyk, Individual Semiosis in Multi-Agent Systems, Transactions on Computational Collective Intelligence VII. Lecture Notes in Computer Science 7270, pp. 164-197 (2012).

[22] J. Nuyts, Epistemic modality, language, and conceptualization: A cognitive-pragmatic perspective, Amsterdam: John Benjamins Publishing Co., 2001.

[23] A. Pieczyńska-Kuchtiak, Experience-based learning of semantic messages generation in resource-bounded environment, Systems Science 30(2), pp. 115-132 (2004).

[24] A.S. Rao, M.P. Georgeff, Modelling rational agents within a BDI-architecture., Proc. KR '91, USA, San Mateo, pp. 473484, 1991.

[25] D. Roy, Semiotic schemas: A framework for grounding language in action and perception., Artificial Intelligence 167(1-2), pp. 170-205 (2005).

[26] G. Skorupa, Grounding of modal conditionals in agent systems, PhD Thesis, Wrocław: Wrocław University of Technology, 2013.

[27] M. Singh, Multiagent systems: a theoretical framework for intentions, know-how, and communications., SpringerVerlag, Heilderberg, 1994.

[28] F.J. Stachowiak, Tracing the role of memory and attention for the meta-informative validation of utterances, [in] Metainformative Centering in Utterances: Between Semantics and Pragmatics, A. Włodarczyk, H. Włodarczyk (eds), John Benjamins Publishing Co., Amsterdam, pp. 121-142, 2013.

[29] R. Sun, Symbol grounding: a new look at an old idea., Philosophical Psychology 13(2), pp. 149-172 (2000).

[30] L. Talmy, Toward a Cognitive Semantics, Cambridge, MA: MIT Press, 2000.

[31] G. Underwood, Implicit Cognition, Oxford University Press, 1996.

[32] P. Vogt, Anchoring of semiotic symbols., Robotics and Autonomous Systems 43(2-3), pp. 109-120 (2003).

[33] P. Vogt, The Physical Symbol Grounding Problem., Cognitive Systems Research 3(3), pp. 429-457 (2005).

[34] D. Więcek, R.P. Katarzyniak, Modal equivalences as linguistic summarisation of data resources, [In:] Information Systems Architecture and Technology: Intelligent Information Systems, Knowledge Discovery, Big Data and High Performance Computing, Wrocław University of Technology, Wrocław (Poland), pp. 23-32, 2013. 
[35] A. Włodarczyk, Grounding of the meta-informative status of utterances, [In] A. Włodarczyk and H. Włodarczyk, Eds., Meta-informative Centering in Utterances: Between Semantics and Pragmatics, John Benjamins Publishing Co., Amsterdam, pp. 41-58, 2013.
[36] T. Ziemke, Rethinking Grounding. In: A. Riegler, M. Peschl, von A. Stein (Eds.), Understanding Representation in the Cognitive Sciences., Plenum Press, New York, pp. 177-190, 1999.

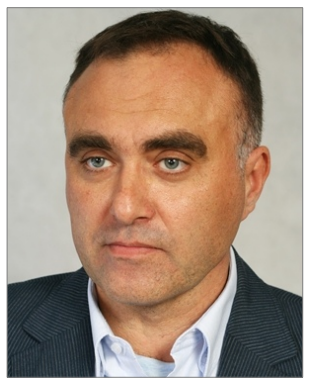

Radosław Katarzyniak Personal Data: born 01 June 1966, Kalisz, Poland; Current Position: Professor of Computer Science, Wrocław University of Technology (WrUT), Faculty of Computer Science and Management, Wrocław, Poland; Head of Computational Semiotics and Interactive Systems Group; Academic Education: 1991: MSc in Computer Science from WrUT; 1999: PhD in Computer Science from WrUT; 2009: D.Sc. (Habilitation) in Computer Science from AGH University of Science and Technology, Poland; Professional Activities: organizing chair of multiple conferences (IEA-AIE'08; International Conference on Collective Computational Intelligence - ICCCI'09, ICCCI'10, ICCCI'11; Asian Conference on Intelligent Information and Database Systems - ACIIDS'09, ACCIDS'10); member of: SMCS Technical Committee on Computational Collective Intelligence and the Technical Committee of KES Focus Group on Agent and Multi-agent Systems; editor and co-editor of monographs on application of computational intelligence methods to knowledge management; guest editor of special issues in international journals on multiagent systems and computational intelligence applications; author of about 150 research journal and conference papers; Current Research Interests: autonomous systems, cognitive linguistics, natural language engineering, knowledge management, computational intelligence.

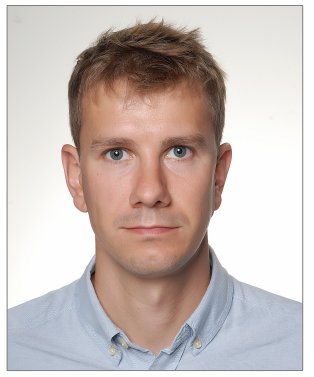

Dominik Więcek Personal Data: born 31 January 1987, Kobyla Góra, Poland; Current Position: PhD Student, Wroclaw University of Technology (WrUT), Institute of Informatics, Wroclaw, Poland; Member of Semantic Systems Engineering Group, WrUT; Academic Education: 2011: MSc in Computer Science from WrUT; Professional Activities: Organising Committee member of international conference ICCCI'12, Senior Software Developer at Applications and Systems Department, Senior IT Assistant at Wroclaw Centre for Networking and Supercomputing, WrUT; Author and co-author of 7 research journal and conference papers; Current Research Interests: artificial and ambient intelligence - agent and multi-agent systems modelling, artificial cognition - semantic communication, symbol grounding, semantic systems engineering, natural and artificial cognition - cognitive semantics, computational cognition and knowledge extraction. 